\title{
Maces et autres noms propres anciens : mise au point linguistique complémentaire
}

\section{S. Chaker}

\section{(2) OpenEdition}

\section{Journals}

\section{Édition électronique}

URL : http://journals.openedition.org/encyclopedieberbere/387

DOI : $10.4000 /$ encyclopedieberbere.387

ISSN : 2262-7197

Éditeur

Peeters Publishers

\section{Édition imprimée}

Date de publication : 29 décembre 2010

Pagination : 4463-4465

ISBN : 978-90-429-2367-6

ISSN : 1015-7344

Référence électronique

S. Chaker, "Maces et autres noms propres anciens : mise au point linguistique complémentaire ", Encyclopédie berbère [En ligne], 30 | 2010, document M06b, mis en ligne le 17 septembre 2020, consulté le 27 octobre 2020. URL : http://journals.openedition.org/encyclopedieberbere/387 ; DOI : https://doi.org/10.4000/encyclopedieberbere.387

Ce document a été généré automatiquement le 27 octobre 2020.

(c) Tous droits réservés 


\section{Maces et autres noms propres anciens : mise au point linguistique complémentaire}

\section{S. Chaker}

1 Le présent volume de l'EB comporte un nombre élevé de notices consacrées à des noms propres de peuples berbères anciens, ou à des toponymes et anthroponymes, antiques ou médiévaux. L'étude de la formation et de la signification (éventuelles) de ces noms est une démarche légitime et nécessaire qui, a priori, peut être éclairante sur certains aspects socioculturels des mondes berbères anciens : contacts avec les autres cultures et langues en présence, conceptions sociales et croyances... En attendant la future notice "Onomastique ", qui examinera de façon approfondie ce sujet et les problèmes qu'il pose, il parait important de souligner que l'approche des matériaux onomastiques berbères anciens est un terrain semé d'embûches qui nécessite à la fois une méthode stricte et beaucoup de prudence.

De nombreuses hypothèses ou rapprochements, qui circulent souvent depuis un siècle dans les différents domaines de spécialités des études nord-africaines, apparaissent extrêmement fragiles ou purement spéculatifs du point de vue de la linguistique berbère. Même lorsque les auteurs en sont d'éminents savants comme le grand Stéphane Gsell ! Il en va ainsi du rapprochement, classique chez les historiens, entre les «Macénites » et les « Miknasa » médiévaux (voir note de S. Chaker sous « Macenites »). Et ne parlons pas de toutes les «étymologies populaires » reprises et prises pour argent comptant par les géographes et historiens contemporains (on en trouvera un exemple sous « Moulouya ; voir aussi la note de S. Chaker).

En fait, en matière d'onomastique berbère et nord-africaine ancienne, il convient de rappeler un certain nombre d'évidences, trop souvent oubliées :

4 On ne dispose que rarement de la version berbère des noms propres. Nos sources sont égyptiennes, puniques, grecques, latines ou, pour le Moyen âge, arabes. Or, dans ces situation de contacts linguistiques, les noms propres indigènes sont toujours plus ou moins profondément déformés par la ou les langues relais, pour une raison linguistique 
évidente et universelle: les systèmes phonologiques des langues en contact peuvent être très différents, dans leurs inventaires de phonèmes, dans leurs structures syllabique et accentuelle et, la (ou les) langues(s) relais va (vont) automatiquement soumettre au filtre de son système phonologique propre les noms indigènes. C'est ainsi que le chef franc [Xlodvik] s'appelle en français « Clovis »...

De plus, dans le cas berbère, quand on travaille à partir d'une forme grecque et/ou latine, il se peut bien souvent qu'elle provienne non pas directement du libyque, mais d'un intermédiaire punique : il ne s'agit donc pas du reflet direct d'un modèle indigène, mais de la reproduction d'une forme relais punique, déjà plus ou moins réinterprétée par rapport à l'original! On a ainsi souvent deux, voire trois, filtres linguistiques qui se superposent : berbère > punique + grec/latin.

6 En conséquence, on doit être extrêmement prudent dans tout rapprochement et toute tentative d'étymologie, fondés sur des matériaux aussi incertains. Si l'on ne connaissait pas l'original arabe ( $a l-j a z a \bar{a} i r)$, on serait bien en peine d'expliquer ou d'analyser le nom d'Alger ; idem pour Bougie, dont l'original berbère Bgayet est passé par l'arabe B(i)jāya.

7 Au plan de l'analyse étymologique des formes et des rapprochements que l'on peut avancer, il convient également de rappeler quelques exigences de méthode. Certes, en phonétique générale, on sait que les sons des langues peuvent connaitre au cours de l'évolution linguistique les avatars les plus étranges, les plus inattendus! Le non spécialiste peut même avoir l'impression que toutes les évolutions et tous les rapprochements sont possibles; et que l'on peut toujours trouver, dans telle ou telle langue, des exemples d'évolutions ou correspondances phonétiques les plus improbables. Sur cette pente, on peut vite être tenté de rapprocher n'importe quoi de n'importe quoi et, subséquemment, de considérer la possibilité qui en existe en phonétique générale (ou dans telle ou telle langue) comme un élément de démonstration. Il est évident que ce type d'approche ne peut être acceptée du point de vue de la linguistique historique, domaine parfaitement cadré de la linguistique, et dont les méthodes constituent, depuis fort longtemps, l'un des «noyaux durs » de la discipline.

8 En matière d'hypothèse étymologiques (onomastique ou autres), la saine méthode exige que l'on on se positionne dans une langue particulière, au maximum dans un domaine linguistique, avec ses contraintes et ses règles phonologiques et morphologiques propres. C'est ce qui permet, par exemple, d'exclure le rapprochement « Macenites » / « « Miknasa », incompatible avec les règles de formation du nom en berbère.

9 Par ailleurs, au plan phonétique, tout rapprochement n'est recevable que s'il n'est pas isolé, c'est à dire qu'à la condition que l'on puisse établir une correspondance sur plusieurs cas analogues, dans le même type de contexte. Pour pouvoir légitimement rapprocher [m-] de [b-] ou de [w-] en position initiale, et mettre en relations des noms de peuples qui présentent ces sons à leur initiale, il faut, au minimum, fournir une série d'exemples qui illustrerait une telle correspondance, et permettrait de formuler l'hypothèse d'un traitement régional ou diachronique [m- $\leftrightarrow \mathrm{b}-\leftrightarrow \mathrm{w}-]$. Sans une telle série, le linguiste considèrera l'hypothèse comme tout à fait gratuite.

On restera donc réservé sur les rapprochements évoqués par R. Rebuffat ci-dessus dans le premier paragraphe de la notice "Maces", rapprochements qui ne peuvent être reçus que comme des hypothèses à explorer. 
11 Du point de vue de la grammaire berbère, on ne peut parler de manière trop affirmative d'un « radical MK- » dans la mesure où en berbère le $m$ - initial est très souvent un morphème dérivationnel, nominal (nom d'agent, nom d'action, adjectif) ou verbal (marque de moyen/passif/réciproque). Autrement dit, dans une séquence $M K x$, la racine a de bonnes probabilités d'être ${ }^{*} K x$. Ce qui a d'ailleurs une implication phonétique immédiate : il est quasiment exclu, si ce $M$ - initial est bien le morphème dérivationnel évoqué ci-dessus, qu'il puisse alterner avec B-, V- ou quoi que ce soit d'autre (en dehors de N-) car ces marques dérivationnelles ont une très grande stabilité $\mathrm{du}$ fait de leur statut grammatical et de leur fréquence dans la formation des mots berbères.

12 De même, la mention de Macurgum, Macurtam, Maqur à propos des Maces paraît inopportune, au moins pour deux raisons :

- le nom de ces divinités berbères anciennes réfère de façon immédiate à une forme verbale quasiment transparente, et tout à fait vraisemblable au plan sémantique, il s'agit de la $3^{\mathrm{e}}$ pers. masc. sing. du thème de prétérit (ou " accompli ») du verbe de qualité imzur, "être grand », ce qui donne maqqur, meqqur, meqq ${ }^{w}$ er, « il est grand».

- Dans Maqur, Macur-, le /r/ fait, en tout état de cause, partie de la racine, ce qui rend encore plus improbable un quelconque lien avec les « Maces».

13 Dans le domaine scientifique, le droit à l'hypothèse ne peut être contesté, mais celui-ci doit rester compatible avec les règles de méthode de la discipline précisément concernée.

\section{INDEX}

Mots-clés : Diachronie, Etymologie, Linguistique, Onomastique 